\title{
Clinical judgment in rheumatoid arthritis. II. Judging 'current disease activity' in clinical practice
}

\author{
J. R. KIRWAN, ${ }^{1}$ D. M. CHAPUT DE SAINTONGE, ${ }^{2}$ C. R. B. JOYCE, ${ }^{3}$ AND \\ H. L. F. CURRE Y' \\ From the ${ }^{1}$ Bone and Joint Research Unit and the ${ }^{2}$ Department of Therapeutics, the London Hospital Medical \\ College London E1 2AD, and the ${ }^{3}$ Medical Department, Ciba-Geigy AG, 4002 Basle, Switzerland
}

SUMmaRY Two rheumatologists made judgments about 'current disease activity' in real patients and 'paper patients' with rheumatoid arthritis. Analysis of each set of judgments provides a model of judgment policy which contains only 3 clinical variables but explains over $94 \%$ of the variance in judgments. The judgment policy models differ markedly from each other and from the clinicians' own perceptions of their behaviour. Judgment policy modelling offers a means of improving co-ordination between clinical investigators within and between centres.

Clinicians differ in the importance they claim to attach to measures of disease activity in rheumatoid arthritis, ${ }^{1}$ but it is not known whether their stated opinions reflect actual practice when making clinical decisions about patients. Using 'paper patients'-a convenient technique developed for presenting standard patient data to several doctors on different occasions ${ }^{1}$ - and judgment analysis-a form of multivariate analysis of the information on which decisions are based ${ }^{2}$ - the present study investigates how clinicians use information to help them make judgments of 'current disease activity' in rheumatoid arthritis. It aims to answer the following questions: (a) How can rheumatologists' decision policies be adequately described or modelled? $(b)$ How do such models of actual behaviour compare with their own view of their policy?

\section{Materials and methods}

Two rheumatologists saw between them 19 patients with RA at routine outpatient consultations over a 2-week period and noted the information that they considered contributed to their assessment of the patient's disease activity. This information was used to construct paper patients as previously described. ${ }^{1}$ Some weeks later 'current disease activity' was assessed in these patients by making judgments on a visual analogue scale (VAS) $100 \mathrm{~mm}$ long marked 'no disease activity' at the left end and 'maximum possible disease activity' on the right. A similar judgment had also been made at the time the clinician first saw the real patient.

Judgment policy models were extracted from the decisions by the method of all possible subsets regression analysis on a computer. ${ }^{3}$ Briefly, all possible combinations (subsets) of the data in the 'paper patients' are correlated with the judgments made by the doctor. The combination which produces the regression line best fitted to the judgments is taken as the model. The relative contribution of each clinical variable (or cue) to the model indicates the importance (or weight) of the cue in question. Here the weight is assessed by calculating the loss of explained variance when each cue in turn is omitted from the regression equation and is thus the 'relative contribution to $\mathrm{R}^{2}$, for that cue. Those cues for which the relative contribution to $R^{2}$ is low could be omitted from the model with little loss of its ability to represent actual practice; on the other hand cues with a high relative contribution are obviously essential elements.

In addition to judging paper patients the clinicians then recorded the importance they attached to the same cues as measures of disease activity by dividing 100 marks between them, higher scores indicating a greater relative weight.

\section{Results}

Although the physicians were given an opportunity to record a large number of clinical variables, in practice they noted only 5 for each of the patients 
Clinical judgment in rheumatoid arthritis. II. 649

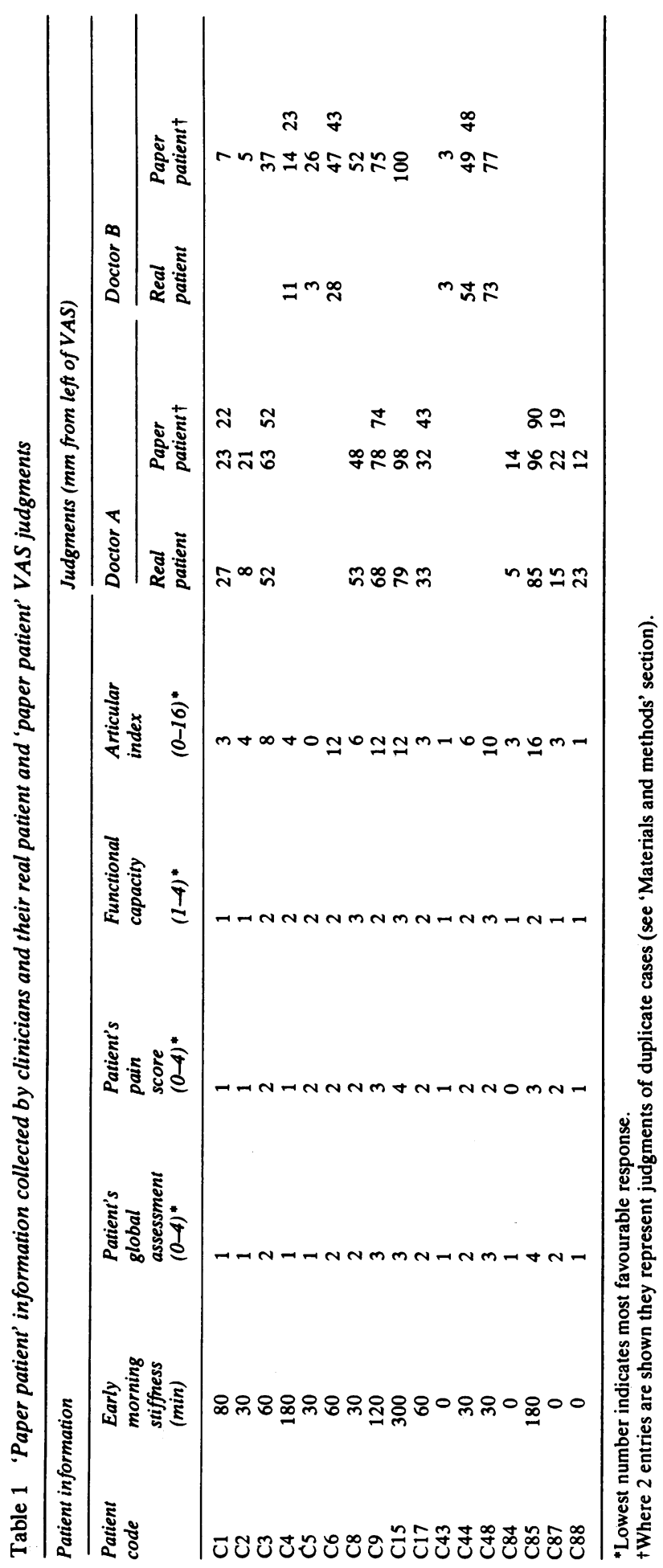


seen: early morning stiffness, patient's global assessment, patient's pain score, functional capacity, and articular index. Details of the data collected are shown in Table 1 together with VAS judgment scores for both real and 'paper' patients and including duplicated judgments for some of the 'paper patients'. There is good agreement between scores for real and the equivalent 'paper' patients when judged by the same clinician, and between the duplicated judgments, as has been observed previously. ${ }^{1}$ The models were based on all the judgments made by each clinician (28 judgments by Doctor $A$ and 21 by Doctor $B$ ), and the extracted policy model equations, together with the proportion of the variance each explains, are:

Doctor $A$

$\mathrm{J}=3.9 \times$ articular index $+9.7 \times$ functional capacity $+5.6 \times$ pain score $-8 \cdot 7$.

$\mathbf{R}^{2}=0.949$.

Doctor B

$\mathrm{J}=\mathbf{2 4} \cdot \mathbf{1} \times$ patient's global assessment $+8.5 \times$ functional capacity $+7 \cdot 8 \times$ pain score -36.7 .

$\mathbf{R}^{2}=\mathbf{0 . 9 3 9}$.

The value of the indices in the equations as shown depends on the units of measurement for each cue. The relative importance of the cues to the judgment policies of the doctors (see 'Materials and methods' section) is shown in Fig. 1 as the relative contribution to $\mathbf{R}^{2}$. The doctors' own scores for the importance of the cues are also shown.

\section{Discussion}

Judgments based on paper patients have been shown to agree closely with face-to-face judgments made on the real patients from whom the data were obtained, ${ }^{1}$ and it is therefore reasonable to assume that the paper policies calculated here model actual practice policies. The policy models contain only 3 cues but explain $95 \%$ and $94 \%$ of the variance in clinical judgments of the 2 physicians. These remarkably high proportions indicate that most of the information required to arrive at the judgments has been included in the models. The surprisingly small number of cues or clinical variables is consistent with other work in the social sciences ${ }^{2}$ and medicine ${ }^{4}$ showing that, in general, very few cues are needed to describe judgment policies adequately. Even so, the policies of the 2 clinicians are quite different. That of Doctor A can be described largely by the contribution of the articular index, whereas for Doctor B the patients' global assessment is most important. Similar results have recently been obtained in diagnosis of the severity of depression by general physicians ${ }^{4}$ and psychiatrists by Fisch et al. ${ }^{5}$ using hypothetical patient data, and were foreshadowed by a brief early report in rheumatology. ${ }^{\circ}$

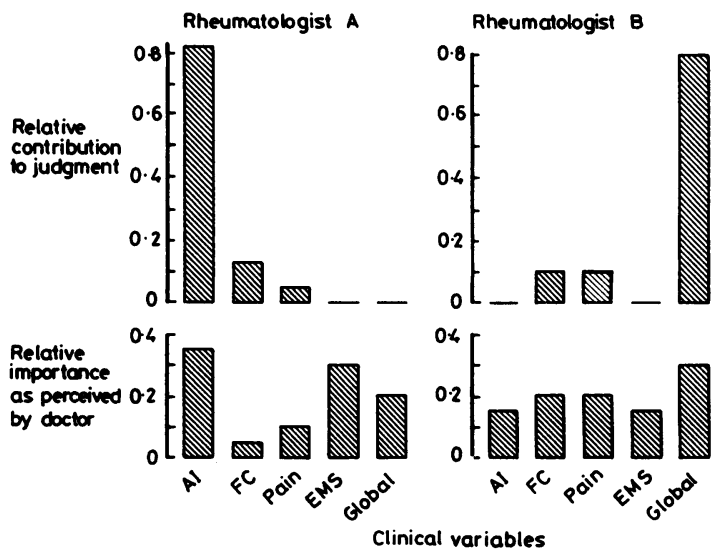

Fig. 1 Actual and perceived contributions of clinical variables to judgments. Actual contributions are the relative contributions to $R^{2}$ derived from the computed judgment models. Perceived contributions are the proportion of 100 marks allocated to each variable by each clinician (see 'Materials and methods' section).

The models derived from the doctors' actual decisions and the relative importance of the cues as seen by the doctors themselves (Fig. 1) also differ. For Doctor A, early morning stiffness and global assessment by the patient appeared strongly in his own perceptions but did not appear at all in the judgment model, and for Doctor B the same was true of pain and early morning stiffness.

Given only the similarity of the doctors' own beliefs about their clinical behaviour we should find it difficult to discover why they differ in their assessments of some patients. The models based on their actual judgments of cases on the other hand allow a better insight into their areas of disagreement. The intention here has not been to specify a 'correct' policy but, after identifying the reasons for disagreement, to make possible rational discussion of their importance and implications.

In this study the 2 clinicians made judgments on many common patients. It is not necessary, however, for judges to review the same cases when comparing computed policy models, only for the subsets of cases to be broadly similar. Within this limitation judgment policies can be compared even if there are no cases in common. In this way the clinical decisions of different investigators-for example, in a multicentre drug trial-can be analysed, and by identifying systematic differences in assessment policies between centres disparate results from similar investigations may be explained and used more effectively.

J. R. Kirwan holds an Arthritis and Rheumatism Council Research Fellowship. D. M. Chaput de Saintonge gratefully acknowledges the support of the Nuffield Foundation. 


\section{References}

1 Kirwan J R, Chaput de Saintonge D M, Joyce C R B, Currey H L F. Clinical judgment in rheumatoid arthritis. I. Rheumatologists' opinions and the development of 'paper patients'. Ann Rheum Dis (same issue).

2 Hammond K R, Stewart T R, Brehmer B, and Steinmann D O. Social judgment theory. In: Kaplan M F, Schwatrz S, eds. Human judgment and decision processes. New York: Academic Press, 1975.

3 Frane J W. All possible subsets regression. In: Dixon $\mathbf{W} J$, Brown M B, Engleman L, eds. Biomedical computer programs, P-series. Berkeley: University of California Press, 1981.
4 Fisch H-U, Hammond $\mathrm{K}$ R, Joyce C R B, O'Reilly $M$. An experimental study of the clinical judgment of general physicians in evaluating and prescribing for depression. $\mathrm{Br} J$ Psychiatry 1981; 138: 100-9.

5 Fisch H-U, Hammond K R, Joyce C R B. On evaluating the severity of depression: an experimental study of psychiatrists. $B r$ J Psychiatry 1982; 140: 378-83.

6 Joyce C R B, Berry H, Chaput de Saintonge D M, Domenet J, Fowler P, Mason R M. Judgment analysis of investigators' assessments: a way to reduce one important source of error in multi-centre trials. In: Fehr K, Huskisson E C, Wilhelmi E, eds. International coordination of drug trials. EULAR Bulletin Monograph 1977: 1 